\title{
Sleeping Disorders Among Children with Down Syndrome: A Cross Sectional Study
}

\author{
Huda Alkawari ${ }^{1 *}$ and AlBandary ALjameel ${ }^{2}$ \\ ${ }^{1}$ Department of Pediatric Dentistry and Orthodontics, College of Dentistry, King Saud University, Riyadh, Saudi Arabia \\ ${ }^{2}$ Department of Periodontics and Community Dentistry, College of Dentistry, King Saud University, Riyadh, Saudi Arabia
}

Submission: September 22, 2021; Published: October 04, 2021

*Corresponding author: Huda Alkawari, Department of Pediatric Dentistry and Orthodontics, College of Dentistry, King Saud University, Riyadh, Saudi Arabia

Abstract

Introduction: Sleep disorders should be addressed on priority as such problems are directly associated with increased risk of neurobehavioral complications and growth impairment. Children are at extreme risk of sleep disorders because of stress, anxiety, mental health condition, hyperactivity, genetic disorders. The prevalence of sleep disorders among children in Saudi Arabia is approximately 18\% among school-going children. This study has aimed to identify the association between sleep disorders and Down syndrome among children in Riyadh, Saudi Arabia.

Method: The children with Down syndrome who were attending daycare facilities or special care centers in Riyadh, Saudi Arabia, were selected for the study. A total of 23 children, ranging in age from 10 to 14, were recruited. To obtain data on demographics and subjective aspects, a self-administered, structured, and validated questionnaire was utilized. SPSS version 17 has been used for data analysis in this study.

Results: The finding revealed that $17.4 \%$ of the children faced apneas; however, only $13 \%$ of the children faced troubled breathing. Furthermore, $4 \%$ of the children used to open their mouth during the day for mouth breathing. $52.2 \%$ of the children reported that it is hard to wake up; $39.1 \%$ of the children reported sleepy per instructor and only $13 \%$ of the children reported a problem with sleepiness. $65.2 \%$ of the children faced nocturnal enuresis.

Conclusion: Sleep disorder or sleep apnea is relatively common among Down syndrome Saudi children. However future studies with larger sample size are required to get more in-depth findings.

Keywords: Sleep Disorders; Sleep Apnea; Down Syndrome; Saudi Arabia

\section{Introduction}

Sleep disorder is considered as a common medical condition among children and is mainly characterized by lack of sleep or disturbed sleep patterns among children. It has been evaluated that sleep disorders should be treated on priority to reduce the risk of neurobehavioral complications along with growth impairment. Children are at extreme risk of sleep disorders due to various reasons DelRosso, Mogavero \& Ferri [1]. Stress, anxiety, mental health condition, hyperactivity, congenital disorders, and impaired diet are considered as certain causes behind sleep disorders among children. Obstructive sleep apnea is relatively common among children as compared to central sleep apnea and complex sleep apnea. Previous studies have also shown the prevalence of sleep disorders among children, suffering from Down syndrome Cardoso, Pompéia \& Miranda [2].

Down syndrome (DS) was firstly explained by John Langdon Down in the year 1866 as a genetic disorder, which is caused by an extra copy of chromosome 21. The prevalence of Down syndrome is approximately 1 in 732 infants within the territories of the United States of America Bull [3]; Almahyawi, et al. [4]. Some of the common complications linked with Down syndrome mainly include intellectual disabilities, a higher risk of heart defects, increased risk of hypothyroidism, and otolaryngologic diseases Fortea, et al. [5]. It has been evaluated that sleep problems and Down syndrome are particularly associated with each other. Children, suffering from Down syndrome, have mainly reported sleep-related issues Korayem, et al. [6]. There is a need to understand if the sleep disorders are left untreated, there is an extreme risk of cardiovascular and neurobehavioral complications along with affected growth Labuhn, et al. [7]. Therefore, it is necessary to screen and manage sleep apnea or disorders on priority. There are certain risk factors, which are needed to be observed while screening sleep apnea. Tonsillar hypertrophy, obesity, and snoring are common risk factors of affected sleep 
among children Aldossary [8]. Similarly, allergic rhinitis, prenatal smoking, premature birth, and poor socioeconomic status are also linked with increased risk of sleep apnea among children Lee, et al. [9]. In regard to these risk factors, children suffering from Down syndrome tend to face sleep disorders.

The prevalence of sleep disorders among children in Saudi Arabia is also higher. It has been evaluated that approximately $18 \%$ of Saudi school children have reported sleep disorders. Moreover, past data has shown that $7.45 \%$ of the parents have reported sleep disorders among their children Alhaddad, et al. [10]. Therefore, it can be said that sleep disorders are prevalent among Saudi children. However, studies from Saudi Arabia are limited that have discussed the issue of sleep disorders among children, suffering from Down syndrome. Though, studies from other regions have reported an increased prevalence of sleep disorders among children, suffering from Down syndrome Alsubie \& Rosen [11]. However, epidemiological data and descriptive studies are also needed in Saudi Arabia to confirm this aspect. Therefore, this study has aimed to identify the association between sleep disorders and Down syndrome among children in Riyadh, Saudi Arabia.

\section{Methods}

\section{Research Design}

The study has used a cross-sectional descriptive-based study design to collect data. The rationale behind using a cross-sectional research design is to determine the prevailing characteristics of sleep disorders among the population of Riyadh, Saudi Arabia. The study has been conducted from June 2020 to May 2021.

\section{Population and Settings}

The study has recruited those children, who were suffering from Down syndrome and were attending daycare centers or special care centers in Riyadh city of Saudi Arabia. During the period of this study, only 23 children availed the services of special care centers and schools to address their complications. Those children were aged between 10 years to 18 years.

\section{Inclusion Criteria and Exclusion Criteria}

Specific criteria for the inclusion and exclusion of the participants have been set earlier to reduce the risk of selection bias. The inclusion criteria included children, suffering from Down syndrome; children attending daycare centers in Riyadh city; and children diagnosed with cytogenetic trisomy 21 . The exclusion criteria included children, who were not suffering from Down syndrome; children with Down syndrome not belonging to Riyadh city; the existence of a compound disability; children with detrimental systemic diseases; and uncooperative children/ parents.

\section{Data Collection and Data Analysis}

A self-administered, structured, and validated questionnaire was used to collect data related to demographics and subjective elements. The questionnaire was completed by parentsorguardians of the recruited child. However, the clinical data were obtained by the trained dentist and one calibrated. The questionnaire is comprised of demographic questions, subjective evaluation of health status, and questions linked with sleeping activities of children. After collecting the data, MS Excel Spreadsheet has been used for data entry purposes. Codes and weights were assigned to responses for better analysis. SPSS version 17 has been used for the analysis of data with $95 \%$ confidence limits and at an alpha level of 0.05 .

\section{Ethical Considerations}

Ethical approval has been taken from the Research and Ethics Committee of the King Khalid University Hospital with IRB registration number E-19-3657. Informed consent has been taken from legal representatives of the children before their participation. Privacy and confidentiality of the data have been strictly maintained.

\section{Results}

The results have been developed through SPSS version 17 using the collected data. Demographic data of the participants have been presented in Table 1 . A total of 23 children, aged 10 years to 18 years, with mothers, aged 34 years to 58 years were recruited in the study. It has been evaluated that around $74 \%$ of the participants were female children $(n=17)$; however, $26 \%$ of the participants were male individuals $(n=6)$. In regard to the age of children, 2 children were 10 years old, 7 children were 11 years old, 5 children were 12 years old, 7 children were 13 years old and 2 children were 14 years old. In regards to the age of mothers, 7 mothers were in the age group of 34 years to 39 years; 6 mothers were belonging to 40 years to 45 years age group and 46 years to 51 years age group respectively; 3 mothers were in the age group of 52 years to 57 years; however, only 1 woman belonged to the age group of 58 years or above. $70 \%$ of the mothers of recruited children were not working; however, $17 \%$ of them were working full time and only $13 \%$ of the mothers were working part-time. $96 \%$ of the mothers were married; however, only 1 mother was divorced.

In regards to the educational level, $39 \%$ of the mothers have completed their education from university; $30 \%$ have completed secondary education; $13 \%$ have completed intermediary education; 9\% have completed primary education; however, 1 mother has completed postgraduate education. Only 1 mother was uneducated. The education of the father has also been recorded in the demographic characteristics. 39\% of the fathers have completed secondary education, whereas $22 \%$ of the fathers have completed university education. $4 \%$ of the fathers were postgraduate and 1 father was uneducated. 2 fathers have completed primary and intermediary education respectively. Lastly, the general health of the children has also been recorded. As per the data, $65 \%$ of the children had good general health; however, $31 \%$ of the children had moderate, whereas 1 child had bad general health. 
Table 1: Demographic characteristics of the participants.

\begin{tabular}{|c|c|}
\hline Variables & $(n=23)$ \\
\hline & n (\%) \\
\hline \multicolumn{2}{|l|}{ Gender } \\
\hline Male & $6(26 \%)$ \\
\hline Female & $17(74 \%)$ \\
\hline Total & $23(100 \%)$ \\
\hline \multicolumn{2}{|c|}{ Child Age in years } \\
\hline 10 & $2(9 \%)$ \\
\hline 11 & $7(30 \%)$ \\
\hline 12 & $5(22 \%)$ \\
\hline 13 & $7(30 \%)$ \\
\hline 14 & $2(9 \%)$ \\
\hline Total & $23(100 \%)$ \\
\hline \multicolumn{2}{|c|}{ Mother's Age in years } \\
\hline 34-39 Years & $7(31 \%)$ \\
\hline 40-45 Years & $6(26 \%)$ \\
\hline 46-51 Years & $6(26 \%)$ \\
\hline 52-57 Years & $3(13 \%)$ \\
\hline 58 and more & $1(4 \%)$ \\
\hline \multicolumn{2}{|c|}{ Mother's Employment Status } \\
\hline Yes, Full time & $4(17 \%)$ \\
\hline Yes, Part time & $3(13 \%)$ \\
\hline Not working & $16(70 \%)$ \\
\hline \multicolumn{2}{|c|}{ Mothers by marital status } \\
\hline Married & $22(96 \%)$ \\
\hline Divorced & $1(4 \%)$ \\
\hline \multicolumn{2}{|c|}{ Parents by educational level } \\
\hline \multicolumn{2}{|c|}{ Mother's Education } \\
\hline Uneducated & $1(4 \%)$ \\
\hline Primary & $2(9 \%)$ \\
\hline Intermediary & $3(13 \%)$ \\
\hline Secondary & $7(30 \%)$ \\
\hline University & $9(39 \%)$ \\
\hline Postgraduate & $1(4 \%)$ \\
\hline \multicolumn{2}{|c|}{ Father's Education } \\
\hline Uneducated & $1(4 \%)$ \\
\hline Primary & $2(9 \%)$ \\
\hline Intermediary & $2(9 \%)$ \\
\hline Secondary & $9(39 \%)$ \\
\hline University & $5(22 \%)$ \\
\hline Postgraduate & $4(17 \%)$ \\
\hline \multicolumn{2}{|c|}{ Child's General Health } \\
\hline Bad & $1(4 \%)$ \\
\hline Moderate & 7 (31\%) \\
\hline Good & $15(65 \%)$ \\
\hline Total & $23(100 \%)$ \\
\hline
\end{tabular}

After collecting demographic data, the study has further collected data in regard to different items. The first item is related to snoring frequency, which has been recorded by the parents of included children. The results have shown that $91.3 \%$ of the children do not snore usually. Only $4.3 \%$ of the children used to snore usually. Moreover, $21.7 \%$ of the children used to snore regularly. The second item is related to snoring quality. Loud snoring and heavy breathing have been identified as two subitems under this category. The results have shown that $17.4 \%$ of the children snore loudly; however, $30.4 \%$ of the children breathe heavily. The third item is related to breathing problems. Troubled breathing and observed apneas have been identified as two subitems under this category. $17.4 \%$ of the children faced apneas; however, only $13 \%$ of the children faced troubled breathing. The fourth item is related to mouth breathing. Dry mouth on awakening and mouth open during the day are two sub-items under this category. $30.4 \%$ of the children used to open their mouth during the day for mouth breathing. However, $21.7 \%$ of the children reported dry mouth on awakening.

The fifth item is related to daytime sleepiness. Unrefreshed in the morning, hard to wake up, the problem with sleepiness, and the sleepy per teacher are four sub-items under this category. $52.2 \%$ of the children reported that it is hard to wake up; $39.1 \%$ of the children reported sleepy per teacher; $30.4 \%$ of the children reported being unrefreshed in the morning, and only $13 \%$ of the children reported a problem with sleepiness. The sixth item is related to hyperactivity and inattention. Difficulty in organizing, easily distracted, fidgets, does not listen, on the go, and interrupts are six sub-items under this category. The majority of the children have negatively answered against each item. The results have shown that only $8.7 \%$ of the children do not listen; $4.3 \%$ of the children faced difficulty in organizing; $43.5 \%$ of the children were easily distracted; $26 \%$ of the children faced interrupts, and $13 \%$ of the children were on the go. The final category is related to other symptoms. Nocturnal enuresis, delayed growth, morning headache, and obesity are four sub-items under this category. $65.2 \%$ of the children faced nocturnal enuresis, $39 \%$ of the children were facing obesity; only $4 \%$ of the children used to face morning headaches; however, delayed growth has been reported among $8.7 \%$ of the children (Table 2). Despite that, there is a high percentage of children, who snore while sleeping, but such snoring does not take more than half a period of sleeping according to 91.3\% of the sample with (Mdn. = 2). In addition, $95.7 \%$ of the parents mentioned that they did not observe that their children snore when they are awake (Mdn. =2).

\section{Discussion}

From the above results, it has been evaluated that children, suffering from Down syndrome, were also suffering from obesity and nocturnal enuresis. Also, most of the children used to get distracted easily due to Down syndrome. Moreover, some of the children faced difficulty waking up and were also unrefreshed in the morning. This shows that some of the children used to 
face sleep problems at night that usually affect their freshness in the morning. However, the majority of the children did not face problems with sleepiness. The results have shown different aspects of sleep that have been affected by the current oral health status of children. For example, $60.9 \%$ of children used to snore at sleeping with (Mdn. = 1); $65.2 \%$ of the children used to wake up at night to go the bathroom with (Mdn. = 1); $52.2 \%$ of the children used to sleep with an opening mouth with (Mdn. $=1$ ); and $52.2 \%$ of the children believed that it is difficult to wake up in the morning. Therefore, it can be said that sleep apnea or sleep disorders have been reported among some children of the recruited sample, suffering from Down syndrome.

Table 2: Clinical Problems among Children.

\begin{tabular}{|c|c|c|c|c|c|}
\hline & Item & Yes (\%) & No (\%) & Don't know (\%) & Median \\
\hline \multicolumn{6}{|c|}{ Snoring Frequency } \\
\hline 2 & Usually snores & 4.3 & 91.3 & 4.3 & 2.00 \\
\hline 3 & Always snores & 21.7 & 78.3 & - & 2.00 \\
\hline \multicolumn{6}{|c|}{ Snoring Quality } \\
\hline 4 & Snores loudly & 17.4 & 73.9 & 8.7 & 2.00 \\
\hline 5 & Heavy breathing & 30.4 & 65.2 & 4.3 & 2.00 \\
\hline \multicolumn{6}{|c|}{ Breathing Problems } \\
\hline 6 & Trouble breathing & 13.0 & 87.0 & - & 2.00 \\
\hline 7 & Observed apneas & 17.4 & 82.6 & - & 2.00 \\
\hline \multicolumn{6}{|c|}{ Mouth Breathing } \\
\hline 17 & Mouth open during the day & 30.4 & 69.6 & - & 2.00 \\
\hline 18 & Dry mouth on awakening & 21.7 & 65.2 & 13.0 & 2.00 \\
\hline \multicolumn{6}{|c|}{ Daytime Sleepiness } \\
\hline 23 & Unrefreshed in morning & 30.4 & 69.6 & - & 2.00 \\
\hline 24 & Problem with sleepiness & 13.0 & 87.0 & - & 2.00 \\
\hline 26 & Sleepy per teacher & 39.1 & 60.9 & - & 2.00 \\
\hline 28 & Hard to wake up & 52.2 & 47.8 & - & 1.00 \\
\hline \multicolumn{6}{|c|}{ Inattention/hyperactivity } \\
\hline 34 & Does not listen & 8.7 & 91.3 & - & 2.00 \\
\hline 36 & Difficulty organizing & 4.3 & 91.3 & 4.3 & 2.00 \\
\hline 39 & Easily distracted & 43.5 & 52.2 & 4.3 & 2.00 \\
\hline 41 & Fidgets & - & 95.7 & 4.3 & 2.00 \\
\hline 45 & On the go & 13.0 & 82.6 & 4.3 & 2.00 \\
\hline 49 & Interrupts & 26.1 & 65.2 & 8.7 & 2.00 \\
\hline
\end{tabular}




\begin{tabular}{|c|c|c|c|c|c|}
\hline \multicolumn{5}{|c|}{ Other Symptoms } & - \\
\hline 13 & Nocturnal enuresis & 65.2 & 34.8 & 4.3 & 2.00 \\
\hline 29 & Morning headache & 4.3 & 91.3 & 4.3 & 2.00 \\
\hline 30 & Delayed growth & 8.7 & 87.0 & 4.3 & 2.00 \\
\hline 31 & Obesity & 39.1 & 56.5 & & 2.00 \\
\hline
\end{tabular}

Dosier, Vaughn \& Fan [12] have conducted a comprehensive study based on meta-analysis to identify the prevalence of sleep apnea among children, suffering from Down syndrome. The study has shown that sleep apnea is highly prevalent among children with Down syndrome. Moderate to severe sleep problems are prevalent in the younger age. The study has included 18 past studies, which have collected primary data from different regions. The study has also mentioned that sleep disorders are common among children, suffering from Down syndrome. In this way, it can be said that this study has supported the results of the current study. Another study has been conducted by Fan et al. [13], which has made its focus on the assessment and evaluation of sleep apnea among children suffering from Down syndrome.

The study has shown that patients with Down syndrome are at risk of sleep apnea or sleep disorders. However, age is a major factor that needs to be identified. The findings of the study have mentioned that sleep apnea was common among children, suffering from Down syndrome. Sleep disorders tend to get intensified with the growing age. Therefore, it can be said that this study has also supported the outcomes of the current study. Horne et al. [14] has also supported the findings of the current study. This study has identified sleep disorders among children, suffering from Down syndrome along with breathing problems. It has been evaluated that sleep problems are common among children with Down syndrome. However, breathing problems are relatively not so common among children. There is a need to identify other factors; however, cardiovascular complications and behavioral issues are common due to Down syndrome, which may be triggered by sleep apnea or sleep disorders. Therefore, it can be said that sleep apnea or sleep disorders are common among children, with Down syndrome.

Ingram et al. [15] has mentioned the success rate of tonsillectomy to reduce the risk of sleep apnea among children with Down syndrome. It has been evaluated that the rate of sleep disorders is high among children, suffering from Down syndrome. Therefore, the procedure of tonsillectomy will be helpful to overcome the breathing issues, which will also result in better sleep patterns. Such findings have shown that breathing problems exist among some children, who may go through tonsillectomy for better breathing. In this way, the study has also supported the findings of the current study. A recent study has been conducted by Chawla et al. [16], who identified the rate of sleep problems among children with Down syndrome in Australia. In Australia, the prevalence rate of behavioral and respiratory sleep problems is higher. Therefore, greater awareness is needed to ensure timely management. Moreover, the study has also supported the outcomes of the current study and further mentioned that sleep problems can easily be witnessed among children, suffering from Down syndrome. Another recent study has been conducted by Fuca et al. [17], which has mentioned that Down syndrome can be complicated due to affected sleep patterns among children and adolescents. The rate of sleep disorders is higher among children, suffering from Down syndrome. Moreover, behavioral issues are prominent among children with Down syndrome, which can easily be addressed by early management. For this purpose, there is a need to develop awareness among the parents, who have children, suffering from Down syndrome. In this way, this study has also supported the outcomes of the current study.

\section{Study Limitations and Strengths}

The study has used a cross-sectional study design, which can be considered as a major strength as it is the best study design to determine prevailing characteristics in a specific population. The second strength is data analysis, which has been done appropriately with the help of SPSS. The foremost limitation is related to the limited sample size as only 23 children were recruited; therefore, the results cannot be generalized to the entire Saudi population. Moreover, the second limitation is related to limited duration. The study has gathered outcomes within 1 year. However, the increased duration will also result in the increased recruitment of the children.

\section{Conclusion}

Sleep disorder is a serious medical condition, in which an individual used to suffer from a lack of sleep or disturbed sleep patterns. Past data has shown sleep disorders should be treated on priority to reduce the risk of neurobehavioral complications along with growth impairment. Intellectual disabilities, higher risk of heart defects, increased risk of hypothyroidism, and otolaryngologic diseases are common complications linked with Down syndrome. From the Saudi Arabia perspective, it has been evaluated that approximately $18 \%$ of the Saudi school children used to suffer from sleep disorders. Moreover, $7.45 \%$ of the parents have also reported sleep disorders among their children. The findings have comprehensively identified that some of the 
children used to face sleep problems at night that usually affect their freshness in the morning. Whereas majority of the children did not face problems with sleepiness. The results have shown different aspects of sleep that have been affected by the current oral health status of children. For example, $60.9 \%$ of children used to snore at sleeping; $65.2 \%$ of the children used to wake up at night to go bathroom; $52.2 \%$ of the children used to sleep with an opening mouth, and $52.2 \%$ of the children believed that it is difficult to wake up at morning. Therefore, it can be said that sleep disorders have been reported among some children of the recruited sample, suffering from Down syndrome. Though the results have shown that sleep disorders are prevalent among children, suffering from Down syndrome; however, there is a need to conduct more comprehensive research studies with an increased sample size to better identify the outcomes. At the same time, more cities of Saudi Arabia and the increased duration of the study will also be helpful to get more insights about this topic. Therefore, future studies are further needed to derive out more specific outcomes.

\section{References}

1. DelRosso LM, Mogavero MP, Ferri R (2020) Effect of sleep disorders on blood pressure and hypertension in children. Current Hypertension Reports 22(11): 1-7.

2. Cardoso TDSG, Pompéia S, Miranda MC (2018) Cognitive and behavioral effects of obstructive sleep apnea syndrome in children: a systematic literature review. Sleep medicine 46: 46-55.

3. Bull MJ (2020) Down syndrome. New England Journal of Medicine 382(24): 2344-2352.

4. Almahyawi RA, Alamri AA, Alnefaie MN, Almahyawi RA, Abdu LA (2018) Otolaryngology head and neck related problems among children with Down syndrome: Are the parents of aware of them? The Egyptian Journal of Hospital Medicine 72(8): 4969-4972.

5. Fortea J, Vilaplana E, Carmona-Iragui M, Benejam B, Videla L, et al. (2020) Clinical and biomarker changes of Alzheimer's disease in adults with Down syndrome: a cross-sectional study. The Lancet 395(10242): 1988-1997.
6. Korayem M, Nuha MA, Waleed B, Eman A, Wafa A, et al. (2019) Craniofacial manifestations of Down syndrome: A review of literature. Academia Journal of Scientific Research 7(3): 176-181.

7. Labuhn M, Perkins K, Matzk S, Varghese L, Garnett C, et al. (2019) Mechanisms of progression of myeloid preleukemia to transformed myeloid leukemia in children with Down syndrome. Cancer cell 36(2): 123-138.

8. Aldossary MS (2017) Down syndrome: A review for the dental professionals. IOSR Journal of Dental and Medical Sciences (IOSRJDMS) 16(7): 61-66.

9. Lee CF, Lee CH, Hsueh WY, Lin MT, Kang KT (2018) Prevalence of obstructive sleep apnea in children with Down syndrome: a metaanalysis. Journal of Clinical Sleep Medicine 14(5): 867-875.

10. Alhaddad MH, Anwer F, Basonbul RA, Butt NS, Noor MI, et al. (2018) Knowledge and attitude towards Down syndrome among people in Jeddah, Saudi Arabia. Proceedings SZPGMI 32(1): 56-65.

11. Alsubie HS, Rosen D (2018) The evaluation and management of respiratory disease in children with Down syndrome (DS). Paediatric respiratory reviews 26: 49-54.

12. Dosier LBM, Vaughn BV, Fan Z (2017) Sleep disorders in childhood neurogenetic disorders. Children 4(9): 82.

13. Fan Z, Ahn M, Roth HL, Li L, Vaughn BV (2017) Sleep apnea and hypoventilation in patients with Down syndrome: Analysis of 144 polysomnogram studies. Children 4(7): 55.

14. Horne RS, Wijayaratne P, Nixon GM, Walter LM (2019) Sleep and sleep disordered breathing in children with down syndrome: Effects on behaviour, neurocognition and the cardiovascular system. Sleep medicine reviews 44: 1-11.

15. Ingram DG, Ruiz AG, Gao D, Friedman NR (2017) Success of tonsillectomy for obstructive sleep apnea in children with Down syndrome. Journal of Clinical Sleep Medicine 13(8): 975-980.

16. Chawla JK, Howard A, Burgess S, Heussler H (2021) Sleep problems in Australian children with Down syndrome: the need for greater awareness. Sleep Medicine 78: 81-87.

17. Fuca E, Costanzo F, Celestini L, Mandarino A, Vicari S (2021) Characterization of Sleep Disturbances in Children and Adolescents with Down Syndrome and Their Relationship with Cognitive and Behavioral Features. International Journal of Environmental Research and Public Health 18(9): 5001.

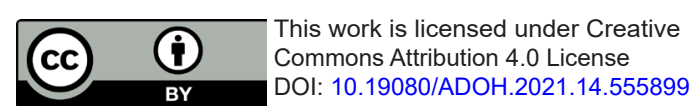

